\title{
Integration of a Manoeuvring Negotiations Module in Driving Support Systems: A Simulator Study
}

\section{Portouli E, Nathanael D* and Marmaras N}

Sector of Industrial Management and Operations Research, School of Mechanical Engineering, Ergonomics Unit, National Technical University of Athens, Greece

*Corresponding author: Dimitris Nathanael, Sector of Industrial Management and
Research Article

Volume 2 Issue 4

Received Date: June 04, 2018

Published Date: June 19, 2018

DOI: $10.23880 /$ eoij-16000160

Operations Research, School of Mechanical Engineering, Ergonomics Unit, National Technical University of Athens, Iroon Politechneiou 9, 15780 Zografou, Greece, Tel: +30-210-772 3938; Email: dnathan@central.ntua.gr

\begin{abstract}
Drivers, when uncertain about others' intent, tend to communicate their own and anticipate others' intent through deliberate communicative acts. Although such communicative interactions among drivers are a crucial component of driving activity they are still not considered in the design of driving support and automation systems. A driving simulator study was conducted, in order to study the effects of integrating a manoeuvring negotiations module, simulating communicative interactions, in three driving support systems. Results from 20 experienced drivers show that participants initiated their manoeuvre sooner, felt more certain and were more in favour when the system provided them with the explicit "consent" of the other involved "driver" than when receiving just a warning according to estimated time to collision. Thus, there are possible benefits from the inclusion of a manoeuvring negotiation module in driving support and automation systems, and further studies should focus on adequately designing "socially interacting" systems.
\end{abstract}

Keywords: Driving Support and Automation Systems; Drivers' Communicative Interactions; Manoeuvring Negotiations Module; Traffic Efficiency; Traffic Safety; Acceptance

\section{Highlights}

- Drivers' communicative interactions are a crucial component of driving activity

- Integration of a manoeuvring negotiations module in support systems was studied

- Drivers started manoeuvre sooner and felt more certain when "consent" was given

- "Socially interacting" driving support and automation systems should be developed

\section{Introduction}

A variety of driving support systems are currently being developed, while recently emphasis is given to cooperative systems which exchange information between vehicles and infrastructure for enhanced situation awareness [1-4]. In order to predict the vehicle trajectories and detect a risk for collision between involved vehicles, such systems typically use motion models based on the laws of physics, possibly integrating interpersonal parameters, such as "preferred" deceleration, the values of which are selected so as to find 


\section{Ergonomics International Journal}

the best compromise between drivers' preferences $[5,6]$. However, drivers' acceptance of such systems is rather limited, their performance being often in discordance to their expectations $[7,8]$, This discordance with drivers expectations is typically being attributed to the systems not counting for the subtleties of human perception and for the important variability in interpersonal characteristics.

Significant research is directed towards the development of fully automated vehicles and it is expected that in the future, vehicles at several levels of automation will co-exist with non-automated vehicles for quite some time. Critical for this coexistence is arguably the capability of automated vehicle systems to interact with drivers in a way that is compatible with the way drivers interact among themselves [9]. Currently in cases of conflict in the motion plans between automated vehicles, either deterministic techniques establishing control rules or stochastic methods are used $[10,11]$. Still in both cases, it is probably impossible to foresee all possible instances of conflicting plans between two or more adjacent automated or non-automated vehicles. Today, in such unforeseen cases, the automated vehicles turn to a central controller, resulting in delays and reduced efficiency of the whole system [12].

With the increasing use of automation systems in cars, human-to-human, machine-to-machine and human-tomachine communication patterns are expected to increase in their significance for car-to-car communication. Recent research has shown that, when drivers predict a possible risky situation in the future trajectories of vehicles, or when other drivers' intent is unclear to them, they tend to communicate in advance their motion plan to other affected drivers [13]. In other words, drivers deliberately seek to interact with other drivers, so as to coordinate towards a safe future motion plan. A typical case is when a Driver A wishes to change lane and there is a constant vehicle flow in the adjacent lane. Driver A switches the direction light and waits. This switching of the direction lights is a communicative act by Driver A. Driver A is in a situation of uncertainty and should confirm the actual intent of other drivers before initiating the lane change manoeuvre. At some point of time, Driver A perceives a Driver B in the adjacent lane flashing headlights and slowing down. This flashing of headlights is a communicative act by Driver B. Sequences of elementary communicative acts of a particular traffic event is hereafter named communicative interactions. Portouli, et al. [13] propose a Linguistic Model of Drivers' communicative Interactions using Austin's typology [14] (Figure 1). Communicative acts and communicative interactions among drivers are a crucial component of driving activity, essential for traffic efficiency and safety. However, communicative interactions may fail due either to non-detection of a communicative act or to its misinterpretation. Such failures can have severe consequences as regards road safety.

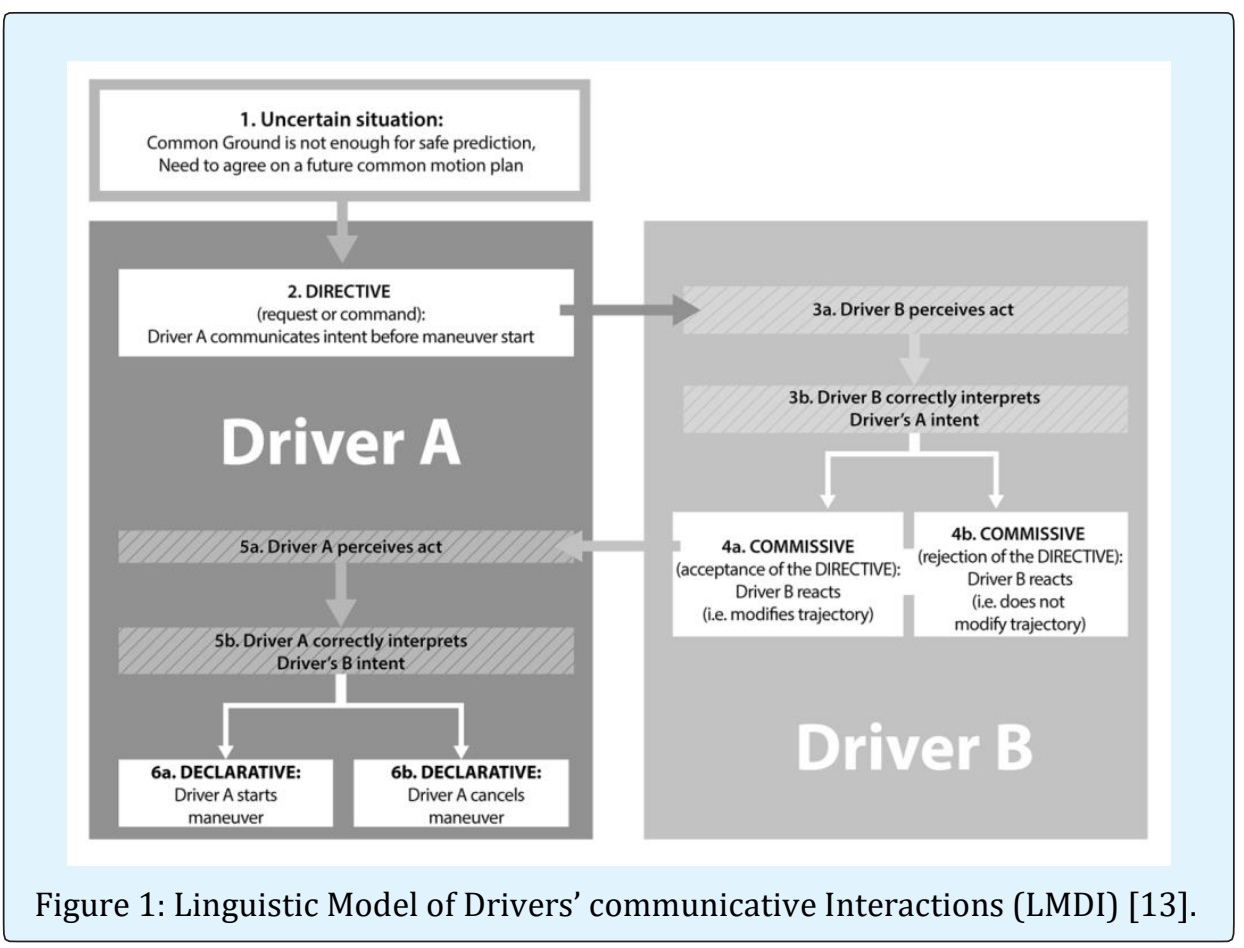

Nathanael D, et al. Integration of a Manoeuvring Negotiations Module in Driving Support Systems: A Simulator Study. Ergonomics Int J 2018, 2(4): 000160. 


\section{Ergonomics International Journal}

Deliberate communicative interactions are not considered in the design of driving support and automation systems, thus the collision risk that these systems estimate does not take into account human drivers' anticipations and deliberate coordination for action. This may be one reason why such systems face limited acceptance by drivers. In the present paper it is investigated whether the integration of a manoeuvring negotiations module, simulating communicative interactions in driving support systems, would be beneficial in terms of traffic efficiency and would therefore be more in accordance to drivers' estimations, as suggested by Portouli, et al. [13]. More specifically, a study using a driving simulatorwas conducted, in order to explore whether such a module (i) would have an effect on traffic efficiency, i.e. drivers would start a manoeuvre sooner and maintain shorter distances to surrounding vehicles during a manoeuvre, (ii) would increase the drivers' certainty about the evolution of the traffic situation and (iii) would perform more in accordance to drivers' expectations and anticipations.

The remainder of the paper is structured as follows: first the setup of the study is presented, including the method, the procedure, the participants' characteristics, the analysis and metrics used. Then the results are presented and discussed. The final section presents the conclusions drawn, the limitations of the study, and directions for further research.

\section{Methods}

\section{Apparatus}

The experiment was conducted on the driving simulator of the Hellenic Institute of Transport (see Figure), which is built around a Smart cabin equipped with sensors. The position of all control levers, windshield wipers, blinker and ignition key and lights switch is transmitted to the driving computer. All operational elements, steering wheel, accelerator pedal, brake pedal, gearshift lever and handbrake lever, provide nature-true force reactions. The sight system includes five largescreens, of $2 \mathrm{~m}$ width each. There is on-screen projection with 2500 ANSI-lumen consumer video projectors. The sound system generates original sounds according to the situation (starter, engine noise, horn, screeching of tires, drive wind, rain, etc.). The vibration device creates nature true vibrations of the car according to the revolutions per minute of the simulated engine. 


\section{Ergonomics International Journal}

In the highway entrance scenario participants were asked to enter the highway starting from a side road. On the right lane of the highway a dense flow of vehicles was programmed to drive at $65 \mathrm{~km} / \mathrm{h}$ with a gap of $15 \mathrm{~m}$, i.e. a time gap of $0.83 \mathrm{~s}$. The instruction given to the participants was to change lane when they would consider it safe to do so. Randomly some of the vehicles flashed headlights without their speed being reduced. After the participants had driven a distance of about $60 \mathrm{~m}$ on the auxiliary lane of the highway, again randomly, one of the vehicles in the right lane flashed headlights and started slowing down with a deceleration of $4 \mathrm{~m} / \mathrm{s}^{2}$.

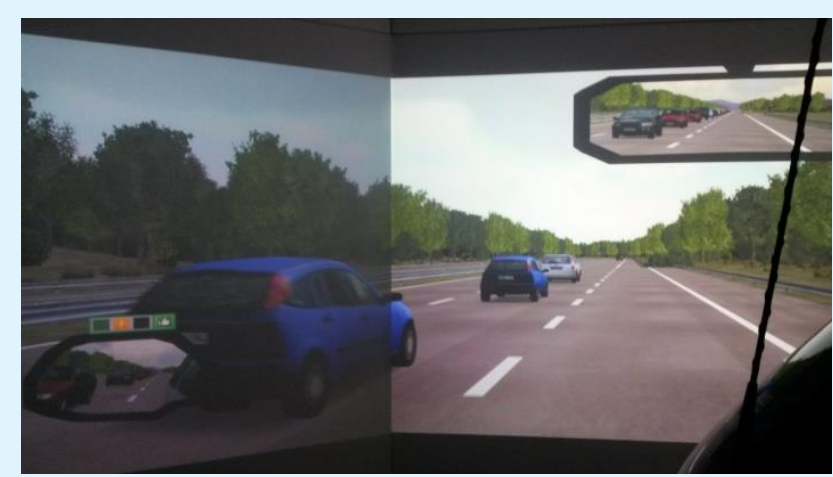

Figure 4: View from the highway entrance scenario.

In the overtaking scenario participants were asked to overtake a slowly-moving heavy vehicle on a rural road. The road had one lane per direction with central lane marking. Due to the large size of the lead vehicle, the visibility was limited. In the opposite lane there was a flow of vehicles programmed to drive at $40 \mathrm{~km} / \mathrm{h}$ at a distance of $80 \mathrm{~m}$. Given the size and speed of the lead vehicle, the overtaking was practically impossible.

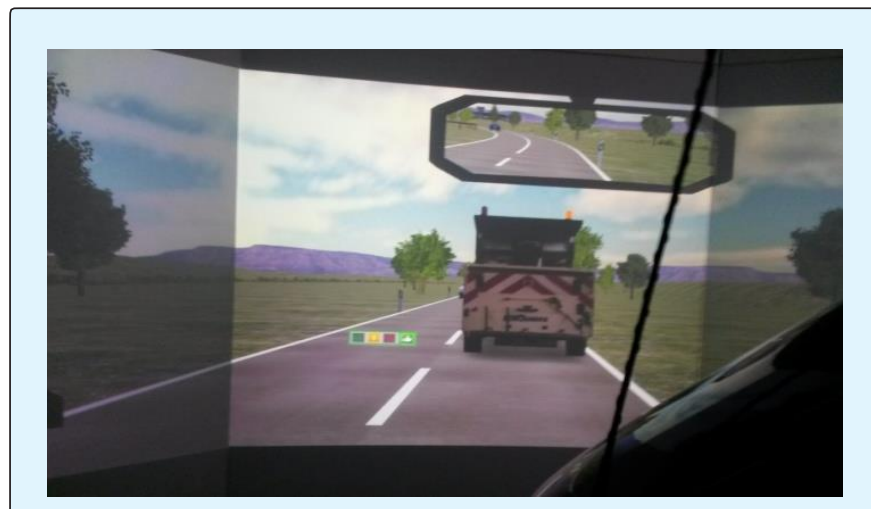

Figure 5: View from the overtaking scenario.
Participants were instructed to overtake when they would consider it safe to do so. Randomly some of the oncoming vehicles flashed headlights without a change in their speed, so as to create uncertainty as regards the "intention of their driver". After participants had closely followed the lead vehicle for 30s, again randomly, one of the oncoming vehicles flashed headlights and started slowing down with a deceleration of $4 \mathrm{~m} / \mathrm{s}^{2}$.

All scenarios ended when the participant had successfully performed the manoeuvre or when a crash had occurred.

\section{Support Systems and Design}

Three driving support systems were simulated, a system warning about crash risk in case of lane change on highways, a system warning about crash risk with the oncoming car in case of left turn on bi-directional urban road and a system warning about crash risk with the oncoming car in case of overtaking on rural road.

Two conditions were simulated for each of the systems. In the first condition ("TTC only"), the warning was provided according to the calculated Time to Collision (TTC) with the other vehicle involved in the manoeuvre. Three risk levels were calculated, high risk when TTC < $1.5 \mathrm{~s}$ for the highway scenario, TTC $<3 \mathrm{~s}$ for the urban scenario and TTC $<15 \mathrm{~s}$ for the rural scenario, medium risk when $1.5<$ TTC $<5$ s for the highway scenario, $3<$ TTC $<6$ s for the urban scenario and $15<$ TTC $<40$ s for the rural scenario and low risk for greater values of TTC. These values were selected in accordance to Campbell, et al. [15], considering a mean driver's reaction time of $0.8 \mathrm{~s}$ and the time required to conduct the manoeuvre, via repetitive tests in the driving simulator. The warnings were presented as visual signs, on the central screen for the urban and rural scenario and close to the left driver's mirror for the highway scenario.

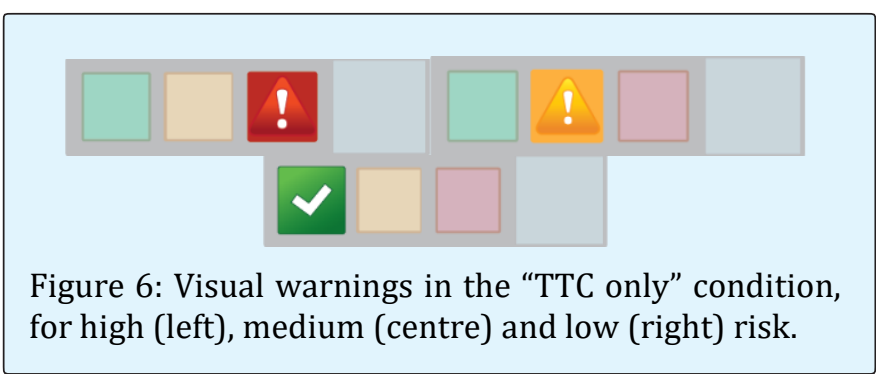

In the second condition ("TTC+intent"), a manoeuvring negotiations module was additionally simulated. This module is essentially a symbolic communication system

Nathanael D, et al. Integration of a Manoeuvring Negotiations Module in Driving Support Systems: A Simulator Study. Ergonomics Int J 2018, 2(4): 000160. 


\section{Ergonomics International Journal}

permitting a driver to communicate his intent, even before physically performing an action. Participants were instructed that as soon as one of the "other drivers" would declare his/her intent to facilitate their manoeuvre through an electronic device in his/her own vehicle, then the system would inform them by additionally displaying an appropriate symbol (Figure 7). In the tests, this symbol was displayed the precise moment the other vehicle started decelerating.

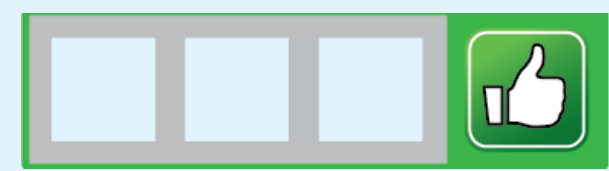

Figure 7: Visual warning for the intent declaration $s$ in the "TTC+intent" condition.

Each participant drove first the urban, then the highway and finally the rural scenario in both "TCC only" and "TCC+intent" conditions in counter-balanced order, to equalize system familiarization effects in the results.

\section{Participants}

The developed scenarios simulated critical driving situations requiring the activation of a driving support system. Therefore it was attempted to engage participants with significant driving experience, experience in driving the specific driving simulator and familiar with driving support systems. This way, participants would need less training before being able to drive the simulator, using in parallel the simulated support systems.

20 people were selected for participation in the study, 13 men and 7 women. The mean age was 41.6 years with a standard deviation of 13.6 years. Participants reported that they had a driving license on average for 18.8 years, with a standard deviation of 13.4 years. 5 people reported that they drive less than $10,000 \mathrm{~km}$ annually, 11 people that they drive $10001-20000 \mathrm{~km}$ annually, 2 people that they drive $20001-30000 \mathrm{~km}$ per year and 2 people that they drive $30001-50000 \mathrm{~km}$ per year. 4 people reported that they are "very experienced" drivers, 8 "experienced drivers" while the other 8 rated themselves as "neither experienced nor inexperienced." As regards driving style, 3 drivers characterized themselves as "conservative" drivers, 10 as "balanced", 5 as "dynamic" and 2 as "very dynamic" drivers. Only 2 of the participants reported having been involved in a road accident in the last 3 years, however without own responsibility. 15 people reported as having "experience driving this simulator" and 5 people as having "significant experience driving this simulator." Many also reported having experience with driving support systems, as shown in the following table.

\begin{tabular}{|c|c|c|c|c|}
\hline & Never heard of it & $\begin{array}{c}\text { I know what it is but I have } \\
\text { never used it }\end{array}$ & $\begin{array}{c}\text { I have some } \\
\text { experience }\end{array}$ & $\begin{array}{c}\text { I have significant } \\
\text { experience }\end{array}$ \\
\hline Driving simulator & & & 15 & 5 \\
\hline Navigation system & 1 & 2 & 13 & 4 \\
\hline Intelligent Cruise Control & 4 & 10 & 4 & 2 \\
\hline $\begin{array}{c}\text { Forward Collision Warning } \\
\text { system }\end{array}$ & 2 & 13 & 3 & 2 \\
\hline $\begin{array}{c}\text { Lateral Collision Warning } \\
\text { system }\end{array}$ & 4 & 12 & 2 & 2 \\
\hline
\end{tabular}

Table 1: Participants' experience with driving simulator and driving support systems (number of answers).

\section{Procedure}

After completing a background questionnaire, participants were given an instructions sheet with the warning symbols. The system's functionalities in the first condition were explained to them. Immediately after that, participants drove all scenarios in this condition and afterwards completed an evaluation questionnaire for the relevant support systems. Then, the functionalities in the second condition were explained. Immediately after that, participants drove all scenarios in the second condition and completed an evaluation questionnaire for the second systems. In the end they completed a short questionnaire comparing the two systems. All questionnaires are included in the Annex. It should be noted that participants were instructed to drive each scenario in the first condition as many times as they wished, until they declared that they felt comfortable with the system.

\section{Analysis}

The simulator logs all dynamic driving parameters relevant to participants' driving behaviour, as well as the location and speed of all surrounding vehicles, at a 


\section{Ergonomics International Journal}

frequency of $30 \mathrm{~Hz}$. The following measures were calculated for each scenario.

\section{$>\quad$ Left Turn in Urban Environment}

- $t_{\text {start }}$ : time (s) from start braking by the oncoming vehicle and start of left turn by the participant

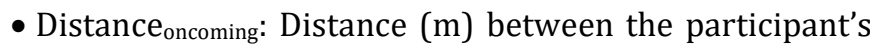
vehicle and the oncoming braking vehicle at $t_{\text {start }}$.

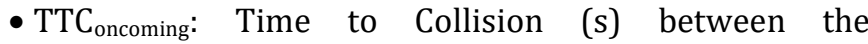
participant's vehicle and the oncoming braking vehicle at $\mathrm{t}_{\text {start. }}$. This is calculated as distance between the two vehicles divided by their relative speed.

- Min_TTC oncoming: Minimum Time to Collision (s) between the participant's vehicle and the oncoming braking vehicle during the whole left turn manoeuvre.

\section{$>\quad$ Highway Entrance}

- $t_{\text {start }}$ : time (s) from start braking by the vehicle in the right highway lane and start of lane change by the participant

- Headwayrear: Time distance (s) between the participant's vehicle and the braking vehicle in the right highway lane at $t_{\text {start }}$. This is calculated as distance between the two vehicles divided by the braking vehicle speed.

- Headwayfront: Time distance (s) between the participant's vehicle and the lead vehicle in the left lane at $t_{\text {start. }}$ This is calculated as distance between the two vehicles divided by the participant's vehicle speed.

- Min_Headwayrear: Minimum time distance (s) between the participant's vehicle and the braking vehicle in the right highway lane during the whole lane change manoeuvre.

\section{Overtaking on Rural Road}

- $t_{\text {start: }}$ Time (s) from start braking by the oncoming vehicle and start of overtaking by the participant

- Distance oncoming: Distance (m) between the participant's vehicle and the oncoming braking vehicle at $t_{\text {start }}$.

- TTC oncoming: Time to Collision (s) between the participant's vehicle and the oncoming braking vehicle at $t_{\text {start. }}$ This is calculated as distance between the two vehicles divided by their relative speed.

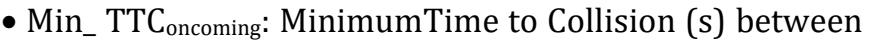
the participant's vehicle and the oncoming braking vehicle during the whole overtaking manoeuvre.

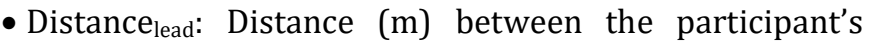
vehicle and the slow lead vehicle at $t_{\text {start }}$.

Mann-Whitney tests were used to study statistically significant differences between the two conditions as regards the above measures. Subjective ratings in the evaluation questionnaires were coded in 5-scale Likert scales, from -2 (most negative rating) to +2 (most positive rating). Differences between the conditions were studied using Wilcoxon signed-ranks tests.'

\section{Results}

\section{Driving Behaviour}

Left Turn on Urban Road Scenario: The time to start the manoeuvre and the TTC to the oncoming vehicle at the time of manoeuvre start were shorter for the "TTC+intent" condition. On the contrary, the distance to oncoming at the time of manoeuvre start and the minimum TTC to the oncoming vehicle during the manoeuvre were longer for the "TTC+intent" condition. Still, these differences were not significant.

\begin{tabular}{|c|c|c|c|c|}
\hline & $t_{\text {start }}(s)$ & Distance $_{\text {oncoming }}(\mathrm{m})$ & TTC $_{\text {oncoming }}$ (s) & Min_TTC $_{\text {oncoming }}$ (s) \\
\hline \multicolumn{5}{|c|}{ "TTC only" } \\
\hline $\mathbf{N}$ & 9 & 8 & 8 & 8 \\
\hline Mean & 3.03 & 7.62 & 4.98 & 0.38 \\
\hline Standard deviation & 1.83 & 7.48 & 6.36 & 1.18 \\
\hline Min & 0.63 & 0.20 & 0.09 & 0.04 \\
\hline Max & 7.07 & 20.31 & 16.36 & 3.27 \\
\hline \multicolumn{5}{|c|}{ “TTC+intent" } \\
\hline $\mathbf{N}$ & 11 & 10 & 10 & 9 \\
\hline Mean & 2.25 & 11.81 & 2.15 & 0.98 \\
\hline Standard deviation & 1.06 & 8.83 & 2.56 & 1.64 \\
\hline Min & 0.60 & 1.77 & 0.27 & 0.07 \\
\hline Max & 4.13 & 24.10 & 8.09 & 3.62 \\
\hline Significance & - & - & - & - \\
\hline
\end{tabular}

Table 2: Effects on driving behaviour-left turn on urban road. 


\section{Ergonomics International Journal}

Highway entrance scenario: The time to start the manoeuvre, the headway to lead vehicle in the right highway lane at the time of manoeuvre start and the minimum headway to the rear vehicle during the manoeuvre were shorter for the "TTC+intent" condition and the differences were significant. The headway to rear vehicle in the right highway lane was also shorter for the "TTC+intent" condition but this difference was not found to be significant.

\begin{tabular}{|c|c|c|c|c|}
\hline & $\mathbf{t}_{\text {start }}(\mathbf{s})$ & Headway $_{\text {rear }}(\mathbf{s})$ & Headway $_{\text {front }}$ (s) & Min_Headway $_{\text {rear }}$ (s) \\
\hline $\mathbf{N}$ & 15 & 15 & 15 & 15 \\
\hline Mean & $\mathbf{3 . 1 8}$ & $\mathbf{0 . 7 7}$ & $\mathbf{1 . 7 5}$ & $\mathbf{0 . 7 6}$ \\
\hline Standard deviation & 1.38 & 0.39 & 1.48 & 0.40 \\
\hline Min & 1.53 & 0.14 & 0.04 & 1.06 \\
\hline Max & 5.44 & 1.63 & 4.10 & 16 \\
\hline $\mathbf{N}$ & 16 & 16 & 16 & $\mathbf{0 . 4 6}$ \\
\hline Mean & $\mathbf{2 . 3 2}$ & $\mathbf{0 . 6 7}$ & $\mathbf{0 . 8 6}$ & 0.46 \\
\hline Standard deviation & 1.41 & 0.37 & 1.02 & 0.04 \\
\hline Min & 0.33 & 0.14 & 0.02 & 1.84 \\
\hline Max & 5.64 & 1.84 & 3.61 & $\mathbf{p}<\mathbf{0 . 0 5}$ \\
\hline Significance & $\mathbf{p}<\mathbf{0 . 0 5}$ & - & $\mathbf{p = 0 . 0 5}$ & \\
\hline
\end{tabular}

Table 3: Effects on driving behaviour-highway entrance scenario.

Overtaking on rural road scenario: The time to start the overtaking and the minimum TTC to the oncoming vehicle during the overtaking were shorter for the "TTC+intent" condition, but the differences were not found to be significant. The TTC to the oncoming vehicle at the time of manoeuvre start was also shorter for the "TTC+intent" condition and the difference was significant $(\mathrm{p}<0.05)$.

\begin{tabular}{|c|c|c|c|c|c|}
\hline & $\begin{array}{l}t_{\text {start }} \\
(s)\end{array}$ & $\begin{array}{c}\text { Distance }_{\text {oncoming }} \\
(\mathrm{m})\end{array}$ & $\begin{array}{c}\text { TTC }_{\text {oncoming }} \\
(\mathrm{s})\end{array}$ & $\begin{array}{c}\text { Min_ TTC oncoming } \\
\text { (s) }\end{array}$ & $\begin{array}{c}\text { Distance } \\
\text { (m) }\end{array}$ \\
\hline \multicolumn{6}{|c|}{ "TTC only" } \\
\hline $\mathbf{N}$ & 19 & 19 & 19 & 19 & 19 \\
\hline Mean & 2.61 & 57.21 & 66.55 & 3.79 & 7.54 \\
\hline Standard deviation & 1.28 & 4.36 & 94.52 & 5.15 & 4.76 \\
\hline Min & 0.53 & 49.41 & 6.90 & 0.43 & 1.52 \\
\hline Max & 5.23 & 66.99 & 271.04 & 24.32 & 16.04 \\
\hline \multicolumn{6}{|c|}{ “TTC+intent" } \\
\hline $\mathbf{N}$ & 19 & 19 & 19 & 17 & 19 \\
\hline Mean & 2.00 & 57.42 & 23.72 & 3.24 & 6.57 \\
\hline Standard deviation & 0.90 & 3.25 & 54.39 & 2.47 & 4.74 \\
\hline Min & 0.87 & 52.16 & 6.22 & 1.29 & 0.30 \\
\hline Max & 4.17 & 62.61 & 247.17 & 11.55 & 18.22 \\
\hline Significance & - & - & $\mathrm{p}<0.05$ & - & - \\
\hline
\end{tabular}

Table 4: Effects on driving behaviour -overtaking scenario.

\section{Subjective Evaluation}

Participants answered that the systems in the "TTC +intent" condition made them drive more safely (mean 0.8 vs 0.6 ), they made them feel safer (mean 1.05 vs 0.95 ) and that the system warnings distracted them less (mean
0.25 vs 0.8$)$. Still, participants answered that the system warnings in the "TTC +intent" condition increased more their workload (mean 0.35 vs 0 ). However, none of these differences were statistically significant. 


\section{Ergonomics International Journal}

\begin{tabular}{|c|c|c|}
\hline Did the system affect your driving? (Made me drive much less safely: -2, Made me drive \\
much more safely: +2) & $0.6(0.99)$ & $0.8(1.2)$ \\
\hline The system warnings made you feel: (Much less safe: -2, Much safer: +2) & $0.95(0.83)$ & $1.05(1)$ \\
\hline $\begin{array}{c}\text { The system warnings: (Increased your workload extremely: -2, Significantly reduced your } \\
\text { workload: +2) }\end{array}$ & $0(0.97)$ & $0.35(1.23)$ \\
\hline $\begin{array}{c}\text { The system warnings distracted your attention when they were not useful? (Surely yes -2, } \\
\text { Surely no: +2) }\end{array}$ & $0.8(1.11)$ & $0.25(1.25)$ \\
\hline
\end{tabular}

Table 5: Comparative usability evaluations of the two conditions, mean (standard deviation).

As regards system learn ability and understandability of symbols and warnings, results are similar for both conditions. Participants gave slightly higher ratings to learnability of the "TTC only" condition (mean 1.65 vs 1.53 ) and to the understandability of its warning icons (mean 1.6 vs 1.47) and slightly higher ratings to the "TTC +intent" condition for the readability of warnings (mean 1.37 vs 1.3 ) and to the understandability of the warning content (mean 1.63 vs 1.53). Still, the differences were not statistically significant.

\begin{tabular}{|c|c|c|}
\hline & "TTC only" & "TTC+ intent" \\
\hline System learning was easy & $1.65(0.49)$ & $1.53(0.51)$ \\
\hline Icons on the screen were legible (good size and contrast) & $1.3(0.92)$ & $1.37(0.76)$ \\
\hline The meaning of the icons was easily understandable & $1.6(0.5)$ & $1.47(0.61)$ \\
\hline I could easily understand the warning given & $1.53(0.7)$ & $1.63(0.6)$ \\
\hline
\end{tabular}

Table 6: Comparative evaluations of system learnability and understandability in the two conditions mean (standard deviation).

Participants reported a positive impression from both types of systems, slightly more positive towards the "TTC+intent" condition (mean 1.11 vs 1.10), however they were slightly more willing to use the "TTC only" condition system (mean 1 vs 0.95), although the differences were not statistically significant.

\begin{tabular}{|c|c|c|}
\hline $\begin{array}{c}\text { Which is your impression from the system you have just experienced? (Very negative: } \\
\text { 2, Very positive: +2) }\end{array}$ & $1.10(0.64)$ & $1.11(0.94)$ \\
\hline $\begin{array}{c}\text { Would you use this system if it was available in the market? (Definitely No: -2, } \\
\text { Definitely Yes: +2) }\end{array}$ & $1.00(0.79)$ & $0.95(1.13)$ \\
\hline
\end{tabular}

Table 7: Attitudes towards the conditions ratings mean (standard deviation).

In the final comparative questionnaire, participants clearly rated the "TTC+intent" condition better, both as regards its accordance to their own estimations (mean 0.47 ) and as regards their feeling of certitude (mean 0.7).

\begin{tabular}{|c|c|}
\hline Question & $\begin{array}{c}\text { Mean (standard } \\
\text { deviation) }\end{array}$ \\
\hline $\begin{array}{c}\text { Which systems warnings were more in accordance to your own estimations of risk? (Definitely of } \\
\text { the "TTC only" condition: -2, Definitely of the "TTC+intent" condition: +2) }\end{array}$ & $0.47(1.39)$ \\
\hline $\begin{array}{c}\text { Which systems warnings made you feel more certain? (Definitely of the "TTC only" condition: -2, } \\
\text { Definitely of the "TTC+intent" condition: +2) }\end{array}$ & $0.7(1.42)$ \\
\hline
\end{tabular}

Table 8: Comparative evaluation of the two conditions.

Participants' comments in favour of the "TTC+intent" condition are: "It provides more certitude for the future motion since there is the agreement of the involved driver", "It supports the communication between drivers in a 


\section{Ergonomics International Journal}

standardized and quick way", "There is a big difference between the two systems, I was absolutely confident that the other driver communicates, while the first system was only indicative", "This system changed my driving behaviour, my feeling of safety and certitude".

Participants' comments not in favour of the "TTC+intent" condition are: "It slightly distracts attention but it helps", "This system is more confusing, the other was simpler", "The other systems warnings were less and the system was less stressful. This system warnings were contradictory to my own estimation". Asked for further clarification, this specific participant responded: "I estimated that I could overtake but I did not see two green symbols, I was seeing a green and an orange one". It seems that this participant expected to see both the agreement of the other "driver" and the green symbol indicating a low-risk TTC value.

One participant did not understand the difference between the two systems. She mentioned: "Both systems are the same in essence".

As regards the visual icons, there were also three comments about their location and it was suggested: "It would be more helpful if the icons were on the central and not on the left mirror". One participant further suggested to avoid using a scale and to present only one relevant symbol each time, to use different schemes and sizes for each level warnings and to use more contrastful schemes and colors for each level. Specifically for the overtaking scenario, another participant suggested that only red or green level warning is useful, as he should take a decision or not, there is no in-between case (i.e. orange warning)

\section{Discussion}

Communicative interactions are essential for coordination in traffic but are not integrated in the design of driving support and automation systems. Only very lately, there are some references to the need for such communication between systems, especially regarding the design of cooperative automation systems in vehicle fleets, which should coordinate locally and negotiate planned maneuvers before performing them [16]. Previous research has suggested the integration of a communicative interactions module, based on a linguistic model, in the design of such systems [17]. The objectives of the present work was to study whether such a system would have an effect on traffic efficiency, whether it would increase the drivers' certainty about the evolution of the traffic situation and whether the system's performance would be more in accordance to drivers' expectations and anticipations.

Some effects on measures relevant to efficiency were found in this study. In more detail, the time to start the manoeuvre was always shorter for the system with the manoeuvring negotiations module, simulating drivers' communicative interactions, and the difference was found to be significant in one of the three simulated scenarios. The time distances to the interacting vehicle in each scenario were always shorter at the time of manoeuvre start in the "TCC+intent" condition, and significant differences were found in two of the three simulated scenarios, meaning that the level of risk according to physics laws at the time of manoeuvre start was higher. No statistical differences were found in the urban scenario, but this may be due to the low number of cases available for analysis owing to technical issues. In any case, in all three scenarios participants initiated their manoeuvre sooner and at conditions of higher objective risk, when there was the "explicit" consent of the other "driver" to their manoeuvre. Participants initiated the manoeuvre in conditions under which they would not initiate it without the explicit "consent" of the other involved "driver", showing that they were relying on the other driver's consent and expected cooperation to their plan. This suggests that participants felt more certain when the system provided them with the explicit "consent" of the other involved "driver" than when the system provided a warning only according to physics laws (i.e. values of TCC).

In support to this, participants were more in favour of the system with the manoeuvring negotiations module, simulating drivers' communicative interactions. They rated this system as being more in accordance to their own risk estimation, they considered that it made them drive more safely, it made them feel safer and more certain, and that the system's warnings distracted them less. These results support the argument that the performance of the system with the manoeuvring negotiations module, simulating drivers' communicative interactions, is more in accordance to drivers' expectations and anticipations [18-20].

Participants answered that this system warnings increased their workload but this may be due to the specific symbol design used in this experiment. It is highly probable that the concurrent warnings by both systems and the usage of green colour in both confused some drivers. This is supported by the higher ratings given to the understandability of the warning icons of the single system but also by the specific comments given. A more 


\section{Ergonomics International Journal}

integrated approach should be studied in the future, meaning that when a driver's acceptance is signaled by the communicative interactions module, then the warning by the standard support system could be suspended, or at least minimized.

Two participants could not discriminate the difference between the two systems; this may be due to the instructions given. More detailed instructions about the functionality of such a system should be provided before its use, although actual familiarization with the system's functionalities will only come with practice.

The results of the present study suggest that there are possible benefits from the inclusion of a manoeuvring negotiations module, in driving support and automation systems, mainly as regards their acceptance by drivers. The latest technological developments and standards for cooperative vehicles may be used as basis for implementing such a module in mixed fleets of automated and manually-driven vehicles. For example, the reference architecture for vehicular ad-hoc networks developed by European Telecommunication Standards Institute could be used. One component of this architecture is the Decentralized Environmental Notification Messages (DENM) protocol. DENM messages are event-driven and application specific warning messages. The DENM protocol could be extended by incorporating handshaketype manoeuvre negotiation protocol involving more than one vehicle, for lane changes or other conflict prone manoeuvres.

The manoeuvring negotiations module could serve as an instantiation of a communicative interactions layer, allowing mixed interactions among human drivers, among automated systems and between drivers and systems, similar to the way that human drivers communicate nowadays.

In the present study, the interaction between drivers was simulated, since there was only one human driver involved. Future studies should involve two or more interacting human drivers and should record and analyse full cycles of communicative interactions on a more naturalistic setting. A study involving a communicative interactions module with natural human interactions may reveal more on the effects of such a module on the human interactions themselves and on the resulting driving behaviour. Furthermore, experiments involving automated vehicles may be conducted, so as to analyse the effect of a communicative interactions module on the interaction patterns between drivers and automated systems and among purely automated systems. Future research should also focus on the design of proper "socially interacting" driving automation systems, as regards the taxonomy of use scenarios, the embedded social rules, the protocol of messages exchange and the content and typology of such messages.

\section{References}

1. Barnard Y, Risser R, Krems J (2011) The Safety of Intelligent Driver Support Systems. Design, Evaluation and Social Perspectives. Ashgate.

2. Grubb G, Jakobsson E, Beutner A, Ahrholdt M (2009) Automatic queue assistance to aid under-loaded drivers. Proc. 16th World Congress on ITS, Stockholm, Sweden.

3. Ibanez-Guzman J, Lefevre S, Mokkadem A, Rodhaim S (2010) Vehicle-to-vehicle communications applied to road intersection safety field results. 13th International IEEE Annual Conference on Intelligent Transportation Systems, Madeira Island, Portugal, pp: 192-197.

4. Fukushima M, Kawata K, Tsukada N (2009) Progress of vehicle-infrastructure cooperative safety support system, DSSS, in Japan. 16 $6^{\text {th }}$ ITS World Congress, Stockholm, Sweden.

5. Schubert R, Richter E, Wanielik G (2008) Comparison and Evaluation of Advanced Motion Models for Vehicle Tracking. In Proceedings of the $11^{\text {th }}$ International Conference of Information Fusion.

6. Tideman M, van der Voort MC, van Arem B (2010) A new scenario based approach for designing driver support systems applied to the design of a lane change support system. Transportation Research Part C: Emerging Technologies 18(2): 247-258.

7. Hegeman G, van der Horst ARA, Hoogendoorn SP (2007) Functioning and acceptance of an overtaking assistant design tested in a driving simulator study. In: Proceedings of the $86^{\text {th }}$ Meeting of the Transportation Research Board, Washington, DC.

8. Lee K, Peng H (2005) Evaluation of automotive forward collision warning and collision avoidance algorithms. Vehicle System Dynamics 43(10): 735751.

9. Hutchins E, Klausen T (2000) Distributed Cognition in an Airline Cockpit. In: Cognition and communication 
at work, Engström Y, Middleton D (Eds.), Cambridge University Press, New York, pp: 15-34.

10. Gregoire J, Bonnabel S, de la Fortelle A (2012) Optimal Cooperative Motion Planning for Vehicles at Intersections. IEEE Intelligent Vehicles Symposium, Spain.

11. Sahin E (2005) Swarm Robotics: From Sources of Inspiration to Domains of Application. In Sahin E, Spears W (Eds.), Proceedings of the SAB 2004 Workshop on Swarm Robotics, July 2004, Lecture Notes in Computer Science, Santa Monica, CA, USA, 3342: 10-20.

12. Mehani O, de la Fortelle A (2007) Trajectory Planning in a Crossroads for a Fleet of Driverless Vehicles. Proceedings of the $11^{\text {th }}$ international conference on Computer aided systems theory (EUROCAST'07), Springer-Verlag, Berlin, Heidelberg, USA, pp: 11591166.

13. Portouli E, Nathanael D, Marmaras N (2014) Drivers' Communicative Interactions: On-road observations and modelling for integration in future automation systems. Ergonomics 57(12): 1795-1805.

14. Austin JL (1962) How To Do Things With Words. Oxford: Clarendon Press.
15. Campbell John L, Richard Christian M, Brown James L, McCallum Marvin (2007) Crash Warning System Interfaces: Human Factors Insights and Lessons Learned. Battelle.

16. de La Fortelle Arnaud, Qian Xiangjun, Diemer Sébastien, Grégoire Jean, Moutarde Fabien, et al. (2014) Network of automated vehicles: The AutoNet2030 vision. 21 $1^{\text {st }}$ World Congress on Intelligent Transport Systems, Detroit, Michigan, USA.

17. Portouli E, Nathanael D, Marmaras N, Papakostopoulos V (2012) Naturalistic observation of drivers' interactions while overtaking on an undivided road. Work 41(S1): 4185-4191.

18. ETSI EN 302665 (2010) Intelligent Transport Systems (ITS); Communications Architecture.

19. ETSI TC ITS TS 102 637-2 (2010) Vehicular Communications; Basic Set of Applications; Part 2: Specification of Cooperative Awareness Basic Service.

20. ETSI TC ITS, TS 102 637-3 (2010) Intelligent Transport Systems (ITS); Vehicular Communications; Basic Set of Applications; Part 3: Specification of Decentralized Environmental Notification Basic Service. 